\title{
Mechanical Stress Modulates SOCS-1 Expression in Human Vascular Smooth Muscle Cells
}

\author{
Marc Dangers Julia Kiyan Karsten Grote Bernhard Schieffer Hermann Haller \\ Inna Dumler \\ Hannover Medical School, Hannover, Germany
}

\section{Key Words}

Mechanical stretch • Vascular smooth muscle cell • Suppressor of cytokine signaling $1 \cdot$ Focal adhesion kinase $\cdot$ Lipid rafts

\begin{abstract}
Background: The growth-promoting effect of mechanical stress on vascular smooth muscle cells (VSMC) has been implicated in the progress of cardiovascular diseases related to elevated blood pressure. The underlying molecular mechanisms are, however, not completely defined. Methods: We have studied primary human aortic VSMC using a model for multilateral stretch. Expression of the suppressor of cytokine signaling (SOCS) family member SOCS-1 and related molecular mechanisms were studied using TaqMan analysis, immunoblotting, protein silencing, specific cell treatment, immunoprecipitation and immunocytochemistry. Results: Mechanical stretch inhibits SOCS-1 mRNA and protein expression. This effect was abolished by cell treatment with methyl- $\beta$-cyclodextrin disrupting lipid rafts and with RGD peptide affecting integrins. Inhibition of integrin interaction with another cellular receptor, urokinase receptor (UPAR), as well as UPAR silencing also abolished stretch-induced SOCS-1 downregulation. Mechanical stretch resulted in UPAR redistribution to lipid rafts and in its colocalization with focal adhesion kinase (FAK). Stretch impairs polyubiq-
\end{abstract}

uitination and proteosomal degradation of FAK leading to FAK upregulation in stretched VSMC. SOCS-1 silencing and inhibition of proteosomal degradation simulate this effect. Conclusion: Our study identifies SOCS-1 as a novel participant involved in the propagation of mechanical stimuli in human VSMC, which might be relevant for the development of cardiovascular diseases.

Copyright $\odot 2010$ S. Karger AG, Basel

\section{Introduction}

Vascular smooth muscle cells (VSMC) are subjected to a dynamic, mechanical environment modulated by pulsatile pressure. Under certain conditions, mechanical stress may initiate pathophysiological adaptations of the vessel wall related to functional changes in VSMC [1]. The underlying molecular and cellular mechanisms that eventually lead to a pathological endpoint remain, however, largely undefined.

The Janus kinase (Jak)/signal transducer and activator of transcription (Stat) pathway is one of the key signaling cascades utilized by VSMC to regulate cellular functions, such as migration and proliferation $[2,3]$, and by cardio-

M.D. and J.K. contributed equally to this work.

\section{KARGER}

Fax +4161306 1234 E-Mail karger@karger.ch www.karger.com

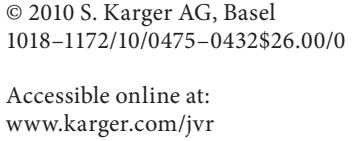

Dr. Inna Dumler

Hannover Medical Schoo

Carl-Neuberg Strasse 1

DE-30625 Hannover (Germany)

Tel. +49511532 2395, Fax +49511532 2713, E-Mail dumler.inna@mh-hannover.de 
myocytes in response to mechanical stress [4]. This pathway is regulated by a family of the suppressors of cytokine signaling (SOCS) that control activation of the Jak/Stat signaling in a feedback loop [5]. Recent studies have demonstrated that beyond controlling the Jak/Stat pathway, SOCS can target several proteins for ubiquitination and proteasomal degradation [6]. Evidence is emerging for the involvement of SOCS proteins in diseases of the immune system [7] and in inflammatory processes [8]. The underlying molecular mechanisms are multifaceted and include regulation of signaling pathways, pro- and antiinflammatory signals as well as targeting signaling intermediates for degradation. The function of SOCS in cellular responses to mechanical signal and in diseases related to elevate blood pressure remains, however, largely unknown, despite its biological and therapeutical significance.

In this study, we examined cellular and molecular mechanisms by which mechanical events are converted into biochemical signals in VSMC. We demonstrate that VSMC respond to mechanical stretch with inhibition of SOCS-1 expression leading to a consequent impaired polyubiquitination and proteasomal degradation of the focal adhesion kinase (FAK). We show that these processes are tightly regulated via RGD-dependent integrins and the urokinase receptor (UPAR), which are organized in a signaling complex in specialized microdomains of cell membrane termed lipid rafts.

\section{Materials and Methods}

\section{Reagents and Antibodies}

Chemicals of high quality commercial grade were purchased from Sigma Aldrich (St. Louis, Mo., USA), Amersham Bioscience Inc. (Little Chalfont, UK), Carl Roth GmbH (Karlsruhe, Germany) and Bio-Rad Laboratories (Hercules, Calif., USA). Mounting medium was purchased from Polysciences Inc. (Warrington, Pa., USA). Integrin blocking RGD (Arg-Gly-Asp) peptides were from Bachem AG (Weil am Rhein, Germany). Protease Inhibitor IX (PI-IX)-AM114 was from Calbiochem (EMD Chemicals Inc. Gibbstown, N.J., USA).

Control siRNA duplexes, uPAR siRNA duplexes and SOCS-1 siRNA duplexes, horseradish peroxidase-conjugated secondary polyclonal antibodies, anti-SOCS-1 polyclonal antibodies (H-93), polyclonal anti-FAK antibodies and monoclonal anti-GAPDH antibodies (6C5) were from Santa Cruz Biotechnology Inc. (Santa Cruz, Calif., USA); anti-ubiquitin polyclonal antibodies were from ENZO Life Sciences (Plymouth Meeting, Pa., USA); anti- $\beta$ tubulin, anti-FAK monoclonal antibodies and polyclonal antiphospho-FAK (Tyr925) antibodies were from Cell Signaling Technology (Beverly, Mass., USA); monoclonal and polyclonal anti-uPAR were from R\&D Systems (Minneapolis, Minn., USA). Alexa 488- and Alexa 633-conjugated fluorescent secondary an- tibodies and Alexa 594-conjugated Rafts labeling kit were from Molecular Probes, Invitrogen (Carlsbad, Calif., USA).

a325 blocking peptide and scrambled controls were kind gifts from Prof. Harold A. Chapman and Dr. Ying Wei (University of California, San Francisco, Calif., USA).

\section{Cell Culture}

Primary human aortic smooth muscle cells were obtained from Promocell (Heidelberg, Germany) and were grown in medium recommended by the suppliers. Cells were plated at a density of 30,000 cells per well onto collagen-coated silicon bottom of 6-well plates (Flexcell International, Hillsborough, N.C., USA) and were grown for up to $72 \mathrm{~h}$ in supplemented growth medium. Medium was changed to growth medium without supplements $24 \mathrm{~h}$ prior to experiments.

\section{Mechanical Stretch Procedure and Cell Treatment}

Cells were subjected to multilateral mechanical stretch $(1 \mathrm{~Hz}$, $15 \%$ elongation) in an incubator at $37^{\circ} \mathrm{C}$ and $5 \% \mathrm{CO}_{2}$ using a Flexercell strain unit FX3000 (Flexcell International, Hillsborough, N.C., USA) for the indicated periods of time as described recently $[9,10]$. Control cells were grown under the same conditions and were put in the identical incubator but not subjected to mechanical stretch.

Blocking of signal transduction pathways by either peptides or methyl- $\beta$-cyclodextrin (MCD) was performed by pretreating cells with respective concentrations of peptides for $1 \mathrm{~h}$ before subjecting the cells to multilateral stretch. Peptides and MCD remained in the medium for the duration of stretch.

\section{Lentiviral Vector Production, Cell Infection, Cell}

Nucleofection

Lentiviral vectors for silencing of UPAR and LacZ were designed and produced as described previously [11]. VSMC were infected in the presence of $8 \mu \mathrm{g} / \mathrm{ml}$ polybrene with viruses at $10^{8}$ $\mathrm{TU} / \mathrm{ml}$ and used for experiments on day 3 after infection. Cell infection rate was about $95 \%$, as controlled by immunoblotting using anti-uPAR antibodies.

For VSMC nucleofection, a human aortic smooth muscle cells nucleofection kit from Amaxa (Lonza Cologne AG, Cologne, Germany) was used in accordance with the manufacturer's instructions. Downregulation of proteins was proved by Western blotting 24-72 h after cell nucleofection and was stable for at least 4-5 days.

\section{Analyses of mRNA Levels by Real-Time PCR}

mRNA was isolated from VSMC using an RNEasy mini kit (Qiagen, Hilden, Germany) according to the manufacturer's instructions. $\beta$-Tubulin served as the reference gene. The sequences of the TaqMan sets read as follows: $\beta$-tubulin: 5'-CAC CAT GAG CGG CGT CA-3'; antisense: 5'-TTC GAA GGT CAG CAT TAA GCT G-3'; probe: 6-FAM-ACC TGC CTC CGT TTC CCG GGTAMRA; SOCS-1: 5'-TTT TTC GCC CTT AGC GTG AA-3', 5'CTC AGG TAG TCG CGG AGG AC-3'.

\section{Western Blotting}

Western blotting was performed as described previously [3]. Chemiluminescent images were captured using VersaDoc-3000 (Bio-Rad Laboratories) and quantified using Quantity One software (Bio-Rad Laboratories). 


\section{Ubiquitination}

Ubiquitination of proteins was evaluated $48 \mathrm{~h}$ following stretch. Cells were lysed in RIPA buffer containing $1 \mathrm{mM} \mathrm{Na}_{3} \mathrm{VO}_{4}$, $1 \mathrm{mM} \mathrm{NaF}, 1 \mathrm{mM}$ PMSF, $10 \mu \mathrm{g} / \mathrm{ml}$ aprotinin, $10 \mu \mathrm{g} / \mathrm{ml}$ leupeptin, and scraped; $0.75-1.5 \mathrm{mg}$ of total protein from the clarified cell lysates was incubated for $3 \mathrm{~h}$ at $4^{\circ} \mathrm{C}$ with anti-FAK antibodies and protein A/G PLUS-agarose, washed 3 times in RIPA buffer and evaluated by Western blotting. To estimate ubiquitinylation of FAK, membranes were probed with antibodies against FAK and ubiquitin.

To inhibit protein proteasomal degradation, cells were pretreated with $10 \mu \mathrm{M}$ of protease inhibitor (PI-IX) for $1 \mathrm{~h}$ prior to stretch.

\section{Immunofluorescent Confocal Microscopy}

Cells labeling for immunocytochemical studies was performed following $30 \mathrm{~min}$ of stretch. Immediately after stretch cells were placed on ice and lipid rafts were labeled using Alexa 594-conjugated cholera toxin B subunit (Rafts labeling kit; Molecular Probes, Invitrogen) according to the manufacturer's instructions. Following the lipid rafts labeling, cells were labeled using polyclonal antiuPAR antibodies, then fixed with $2 \%$ paraformaldehyde in PBS for $15 \mathrm{~min}$ at $4^{\circ} \mathrm{C}$, blocked, and stained with Alexa 488-conjugated secondary antibodies. For uPAR/FAK double staining, cells were fixed immediately after stretch, permeabilized with $0.1 \%$ Triton $\mathrm{X}-100$ in $\mathrm{PBS}$ for $3 \mathrm{~min}$ at $4^{\circ} \mathrm{C}$, and blocked overnight at $4^{\circ} \mathrm{C}$ in $1 \%$ bovine serum albumin in PBS. Cells were labeled with primary antibody ( $2 \mathrm{~h}$ at room temperature) and fluorescently labeled secondary antibodies ( $1 \mathrm{~h}$ at room temperature). Cells were embedded in Aqua-Poly-Mount mounting medium (Polysciences Inc.). After that, the Bioflex membrane was cut with a scalpel and fixed on the microscope slides using the mounting medium. The fluorescence cell images were captured using a Leica TCS-SP2 AOBS confocal microscope (Leica Microsystems, Wetzlar, Germany). All images were taken with oil-immersed $\times 63$ objective, $\mathrm{NA}=1.4$. Images were recorded with detection wavelengths range for Alexa 488 and Alexa 594 double staining to 505-550 and 605-675 nm, respectively; for Alexa 488 and Alexa 633 double staining to 505575 and $645-750 \mathrm{~nm}$, respectively. All images were acquired with a resolution of $1,024 \times 1,024$ pixels.

\section{Statistical Analysis}

All experiments were performed in triplicate. Data are presented as mean \pm SEM of at least 3 independent experiments. Comparisons were made by the two-tailed Student's t test for independent samples or one-way ANOVA and post hoc Scheffé test as appropriate. Differences were considered statistically significant at a value of $\mathrm{p}<0.05$.

\section{Results}

\section{Mechanical Stretch Induces Downregulation of SOCS-1}

To investigate whether the Jak/Stat pathway might be regulated in VSMC in response to mechanical stretch, we addressed members of the SOCS family, which serve as negative modulators of this pathway [5]. We found that

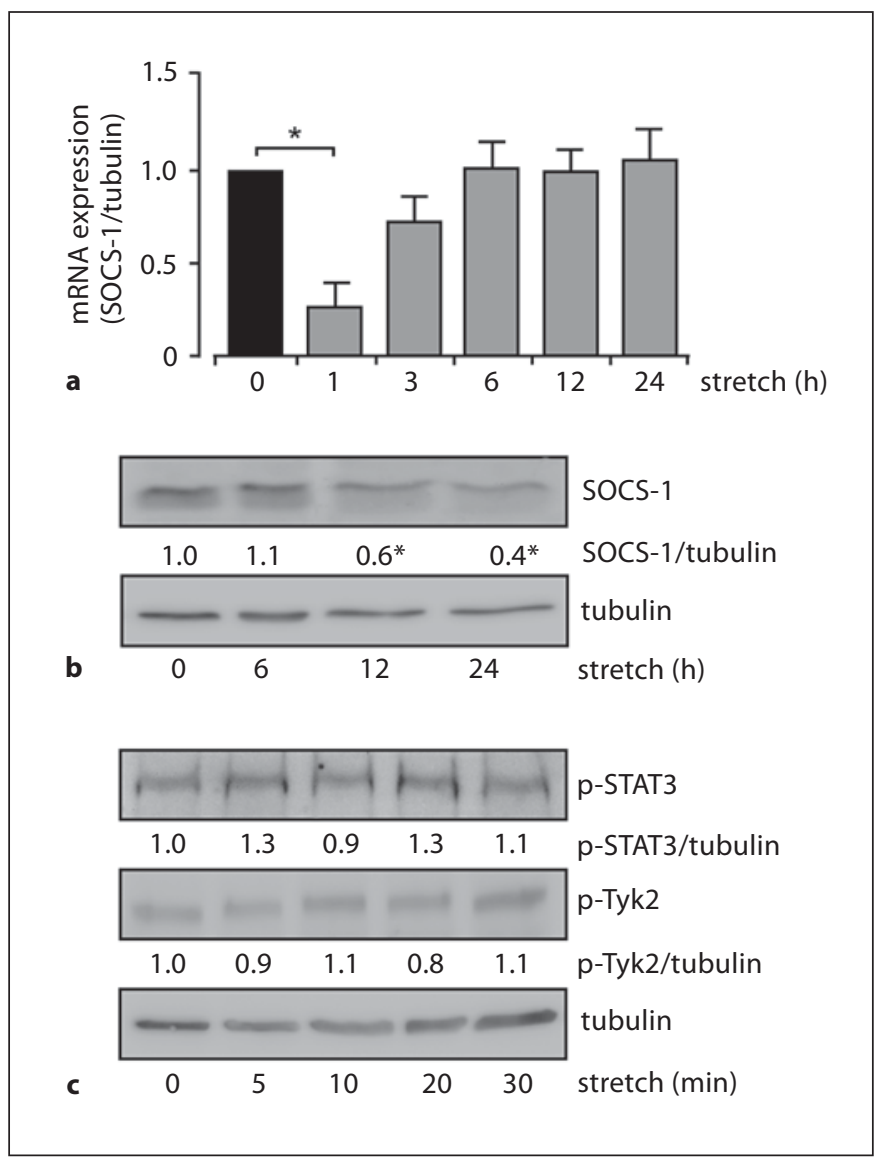

Fig. 1. SOCS-1 is downregulated by mechanical stretch independently of the Jak/Stat pathway. VSMC were seeded onto collagen coated Bioflex 6-well plates and serum starved overnight. Cells were subjected to mechanical stretch for up to $24 \mathrm{~h}$ or were left untreated. a mRNA levels of SOCS-1 were measured by real-time RT-PCR. Values are given as means of three independent experiments relative to untreated control; error bars represent standard deviation. b Samples were analyzed by Western blotting using anti-SOCS-1 antibody. To evaluate equal loading, blots were reprobed with anti-tubulin antibody. c VSMC were seeded onto collagen coated Bioflex 6-well plates and serum starved overnight. Cells were subjected to up to thirty minutes of mechanical stretch or left untreated and harvested by scraping in lysis buffer. Samples were analyzed by Western blotting using anti-phospho-tyrosine Stat 3 or anti-Tyk 2 antibody as indicated. Blots were reprobed with anti-tubulin antibody for a loading control.

expression of SOCS-1 (fig. 1a, b), but not of SOCS-2, SOCS-3 and SOCS-4 (data not shown), was significantly inhibited by cyclic mechanical stretch in a time-dependent manner at the mRNA and protein levels. Surprisingly, this appeared not to be caused by an increase in the Jak/Stat pathway activation. Thus, no changes in phosphorylation of the Janus kinases (Jak1/2 and Tyk2) and 


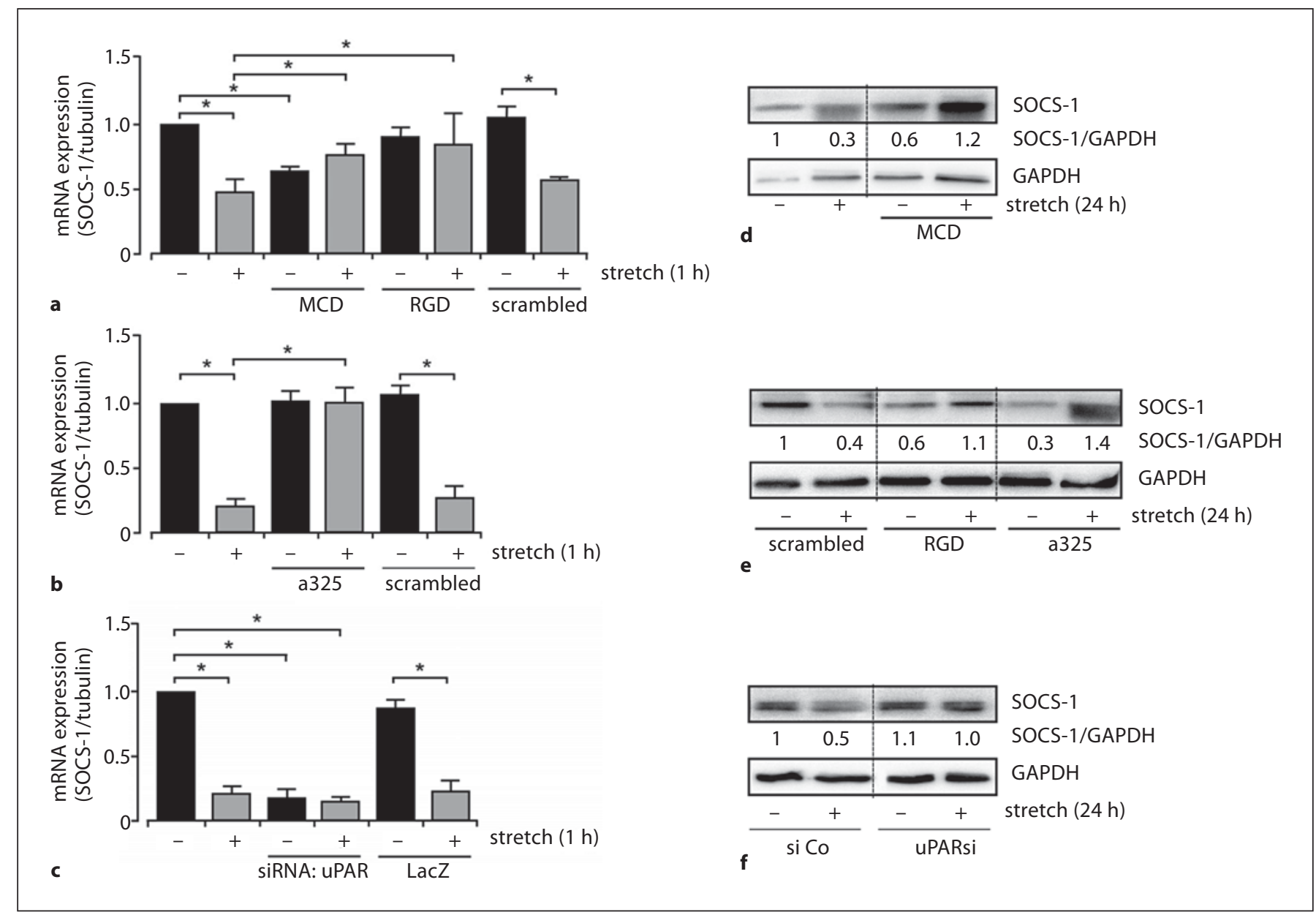

Fig. 2. SOCS-1 downregulation by mechanical stretch is mediated by lipid rafts, integrins and UPAR. VSMC were seeded onto collagen coated Bioflex 6-well plates, serum starved overnight and pre-incubated for $1 \mathrm{~h}$ with the following concentrations of MCD or peptides: $6.5 \mathrm{mg} / \mathrm{ml} \mathrm{MCD;} 25 \mu \mathrm{M}$ RGD peptides; $4 \mu \mathrm{M}$ a325 peptide and scrambled a325 peptide or were left untreated prior to stretch. uPAR expression was downregulated by means of transfection with lentiviral constructs expressing siRNA specific for silencing of uPAR and the LacZ protein as a control (c) or cell nucleofection using uPAR-specific si RNA duplexes (f). After stretch, SOCS-1 expression was analyzed by RT-PCR (a-c) and Western blotting (d-f). For RNA isolation, cells were stretched for $1 \mathrm{~h}(\mathbf{a}-\mathbf{c})$. For Western blot analysis, cells were stretched for $24 \mathrm{~h}$ under similar conditions except that MCD was removed from the medium after $3 \mathrm{~h}$ of stretch, and further $21 \mathrm{~h}$ cells were incubated without MCD. The Western blotting membranes (d-f) were reprobed with anti-GAPDH antibodies for loading control.
Stat proteins (Stat1/3/5) were observed (fig. 1c, shown for Stat 3 and Tyk2). This implies a regulation of SOCS-1 expression in mechanical stretch, which differs from the negative feedback loop regulation of a typical cytokine response. Because the observed stretch-induced decrease in SOCS-1 protein was sustained, whereas downregulation of mRNA was very transient, we examined as to whether or not this effect might be related to SOCS-1 protein degradation. VSMC treatment with the inhibitor of proteasomal degration PI-IX, however, had no effect on the SOCS-1 level (data not shown).
Stretch-Induced SOCS-1 Downregulation Is Mediated by Integrins and $U P A R$, Which Are Organized in Lipid Rafts with FAK

To elucidate the underlying mechanism, we wanted to know which cellular receptor(s) mediates changes in SOCS-1 expression in response to mechanical signal. Integrins have been implicated in propagation of mechanical stretch by changing their conformation or clustering in the cell membrane in response to mechanical stimuli [12]. Mechanical stretch may affect adhesion sites to organize signal molecule-rich specialized membrane mi- 
Fig. 3. Mechanical stretch induces redistribution of uPAR, lipid rafts and FAK. VSMC seeded on Bioflex 6-wells plates were stretched for $30 \mathrm{~min}$. For labeling of lipid rafts (a), cells were placed on ice immediately after stretch and labeled under non-permeabilysing conditions using Alexa 594-conjugated lipid rafts labeling kit (middle panel) followed by uPAR labeling using polyclonal anti-uPAR antibodies (left panel). Then cells were fixed, blocked and incubated with Alexa 488-conjugated secondary antibodies for uPAR visualization. b VSMC were fixed immediately after $30 \mathrm{~min}$ of stretch with 2\% PFA for 10 min on ice. After permeabilization and blocking, cells were labeled with monoclonal anti-uPAR and corresponding Alexa 488-conjugated secondary antibodies (left panel), and anti-FAK and corresponding Alexa 633-conjugated secondary antibodies (middle panel). The right panels show overlay images. Arrows indicate sites of stretch-induced co-localization of UPAR with lipid rafts (a) and FAK (b).

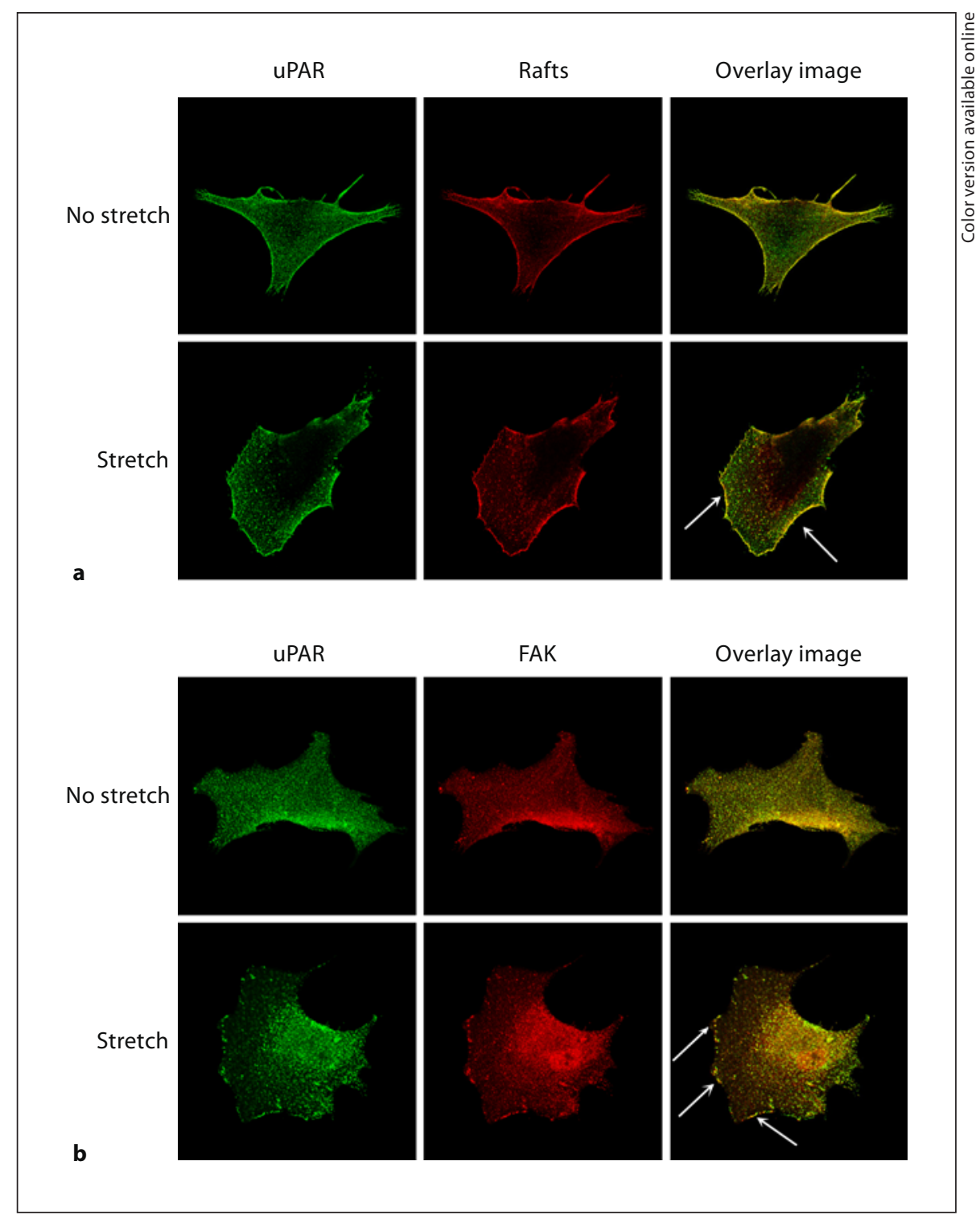

crodomains known as lipid rafts [13] in proximity to integrins, promoting efficient signal transduction.

Therefore, we checked whether lipid rafts and integrins cooperate within the signal transduction machinery of VSMC to affect SOCS-1 expression in response to extracellular mechanical stimuli. Disruption of lipid rafts by cell pretreatment with MCD abolished inhibition of SOCS-1 mRNA expression induced by mechanical stretch. This treatment resulted in a partial inhibition of SOCS-1 expression already in control cells (fig. 2a). Similar results were observed after VSMC pretreatment with integrin-antagonizing RGD peptides, but not with control peptides indicating a contribution of the RGD-dependent integrins to the observed effect (fig. 2a).

It has been demonstrated that integrin functions can be regulated in some cases by another cell surface adhesive receptor, namely the GPI-anchored urokinase-type plasminogen activator receptor $\mathrm{UPAR}$, which is one of the key molecules in the biology of VSMC [14]. To examine whether the regulatory effect of integrins on SOCS-1 expression in response to mechanical stretch might be related to its association with uPAR, VSMC were pretreated with a specific peptide (a325) blocking the uPAR-integrin association. This treatment, but not a control peptide, completely abolished the inhibitory effect of mechanical 

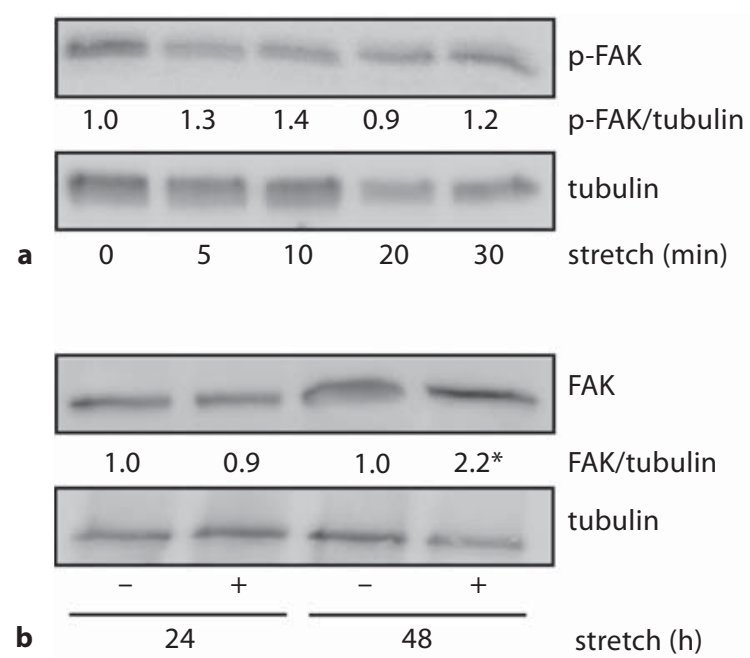

IP: FAK WB: ubiquitin
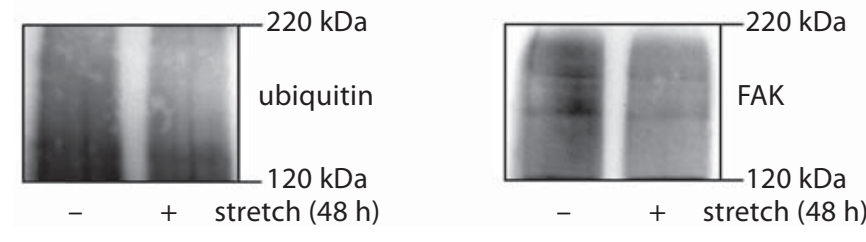

WB: FAK
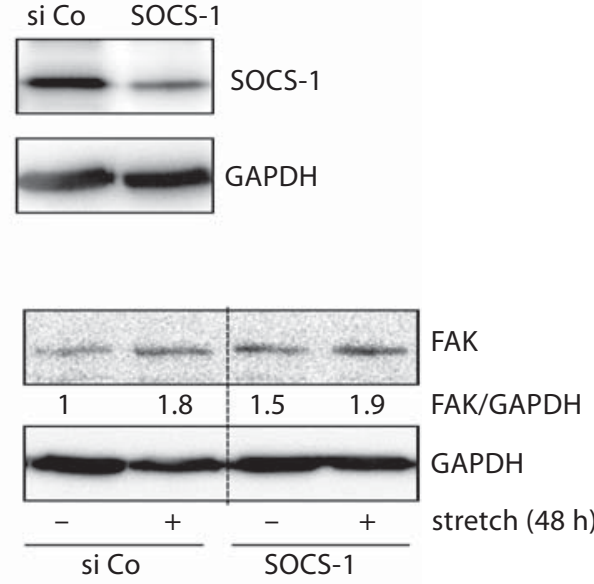

d

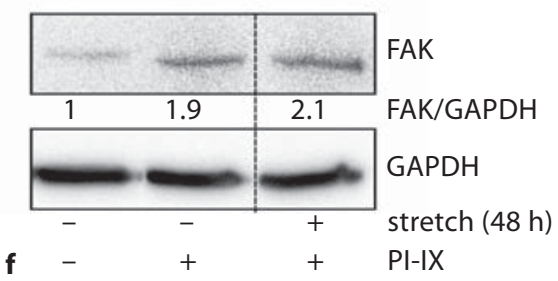

Fig. 4. Protein level and ubiquitination of FAK are regulated by mechanical stretch. VSMC were seeded onto collagen coated Bioflex 6-well plates and serum starved overnight. a Cells were subjected to up to $30 \mathrm{~min}$ of mechanical stretch or left untreated and harvested by scraping in lysis buffer. Samples were analyzed by Western blotting using anti-phospho-FAK antibody. Blots were reprobed with tubulin in order to evaluate equal loading. b Cells were subjected to $2 \mathrm{~h}$ of mechanical stretch or left untreated and harvested by scraping in lysis buffer 24 or $48 \mathrm{~h}$ following stretch. Samples were analyzed by Western blotting with anti-FAK antibody. Blots were reprobed with tubulin in order to evaluate equal loading. c SOCS-1 expression was downregulated in VSMC using nucleofection and SOCS-1-specific siRNA duplexes. The lower

stress on SOCS-1 expression (fig. 2b). To examine this issue further, uPAR downregulation in VSMC was performed by means of an siRNA lentiviral gene transfer. Interestingly, uPAR silencing resulted in an inhibition of SOCS-1 expression already in control cells; mechanical stress did not enhance this inhibition any further (fig. 2c). To substantiate these findings additionally, changes panel shows immunoblotting with GAPDH antibodies as a loading control. d FAK expression after mechanical stretch in VSMC nucleofected with control siRNA duplexes and SOCS-1 siRNA duplexes was estimated after $48 \mathrm{~h}$ of stretch. e Cells were stretched for $48 \mathrm{~h}$ and lysed. FAK was immunoprecipitated and FAK ubiquitination was analyzed by immunoblotting with anti-ubiquitin antibodies (left panel). The right panel shows immunoblotting of lysates with anti-FAK antibodies. $\mathbf{f}$ Cells were pre-treated with $10 \mu \mathrm{M}$ of protease inhibitor (PI-IX) for $1 \mathrm{~h}$ prior to stretch. The amount of FAK was analyzed by Western blotting. The lower panel shows loading control using anti-GAPDH antibodies. ${ }^{*}=\mathrm{p}<$ 0.05 .

in SOCS-1 at the protein expression level were examined (fig. $2 \mathrm{~d}-\mathrm{f}$ ). The results of these experimental settings show that stretch-induced downregulation of SOCS-1 protein was restored after VSMC treatment with MCD, specific peptides and siRNA thus correlating with changes in SOCS-1 mRNA. 
To further analyze lipid raft-related complexes in VSMC subjected to mechanical stretch, confocal microscopy was performed. As shown in figure $3 \mathrm{a}$, in response to cyclic stretch, VSMC underwent changes in their shape and lipid rafts were redistributed in the cell membrane and became significantly enriched in uPAR. We also observed that mechanical stretch induced accumulation in redistributed lipid rafts of the focal adhesion kinase FAK and its colocalization with uPAR (fig. 3b).

Together, these data provide evidence that inhibition of SOCS-1 expression in VSMC in response to mechanical stretch is mediated via association and functional cross-talk of uPAR and integrins linked to lipid rafts. They further suggest involvement in these cellular events of FAK.

Mechanical Stretch Upregulates FAK via Inhibition of Its Polyubiquitination and Degradation That Requires SOCS-1

Because FAK is considered as a primary effector of mechanical stimuli at focal adhesion sites, we were interested in identifying a probable link between the observed stretched-induced enrichment of cell membrane domains in FAK and changes in SOCS-1 expression. Recent reports provide evidence for a regulatory role of SOCS proteins in the FAK pathway [15]. Surprisingly, we did not observe any changes in FAK phosphorylation in VSMC upon mechanical stretch (fig. 4a), whereas FAK protein level was increased (fig. $4 \mathrm{~b}$ ). To examine whether FAK upregulation might be mediated via SOCS-1, we performed SOCS-1 silencing in VSMC (fig. 4c). As shown in figure $4 \mathrm{~d}$, SOCS-1 downregulation resulted in increase of FAK protein already in control cells, which was comparable with those after mechanical stretch; application of mechanical stretch to SOCS-1si-VSMC did not increase this effect any further. These data point to a regulatory role of SOCS-1 in controlling FAK in VSMC.

A growing body of data demonstrate that SOCSs, and in particular SOCS-1, can recruit ubiquitin ligase to a number of proteins and thus can target these proteins for ubiquitination and proteasomal degradation in a SOCS box-dependent manner. However, the relationship between SOCS-1 and the ubiquitin proteasome pathway remains unclear [16]. To investigate whether an increase in FAK protein after mechanical stretch might be a result of changes in SOCS-1-mediated ubiquitination of FAK, protein ubiquitination was studied. As shown in figure 4e, polyubiquitination of FAK was impaired in VSMC subjected to mechanical stretch. Cell treatment with the inhibitor of proteosomal degration PI-IX leads to FAK up- regulation similar to the effect of mechanical stretch (fig. 4f). These data might explain the observed increase in FAK protein in response to mechanical signal as a result of impaired FAK ubiquitination and a subsequent lack of proteasomal FAK degradation. They further suggest that SOCS-1 mediates these processes.

\section{Discussion}

Mechanotransduction plays a critical role in vascular homeostatis. Whereas physiological cyclic stretch maintains vessel wall structure and controls VSMC proliferation, enhanced tensile force occurring in severe hypertension and related diseases initiates dramatic changes in VSMC functional behavior [17]. However, the underlying mechanisms of molecular signaling from the cell surface toward the intracellular machinery are still poorly understood [12]. Our study demonstrates that SOCS-1, a member of the family of suppressors of cytokine signaling, may have a previously unidentified role in mechanotransduction of human VSMC.

We provide evidence that inhibition of SOCS-1 expression in VSMC in response to mechanical stress is mediated via association and functional cross-talk of uPAR and integrins linked to lipid rafts and is independent of the Jak/Stat pathway. The data further suppose a model in which uPAR serves as a mediator, which in a normal situation maintains SOCS-1 expression in an active state. Mechanical stress leads to the abrogation of this control via uPAR association with the RGD-dependent integrins, thus resulting in SOCS-1 downregulation that, in turn, affects polyubiquitination and degradation of FAK (fig. 5).

Integrins serve as an important cellular mechanotransduction system, coupling and integrating mechanical stimuli with intracellular chemical cascades. These dynamic aspects of integrin function are dependent on integrin interactions with its neighbors in the cell membrane and inside the cell [18]. Several findings implicate integrins in cellular responses that are induced by the multifunctional uPA/uPAR system [19]. Our data demonstrate requirement for UPAR-integrin association in VSMC for the observed effect of SOCS-1 downregulation in response to mechanical stretch, as shown in experiments using specific blocking peptides and siRNA. Thus, these results identify a novel function for the cross-talk between integrins and UPAR in VSMC; it remains to be determined whether integrin-uPAR association regulates SOCS protein expression in response 
Fig. 5. Schematic presentation of the proposed model. Under physiological conditions, uPAR maintains SOCS-1 expression in an active state via the RGD-dependent integrins. The complex is organized in lipid rafts. Mechanical stress leads to the abrogation of this control resulting in SOCS1 downregulation and to SOCS-1-directed impaired polyubiquitination and proteosomal degradation of the focal adhesion kinase.

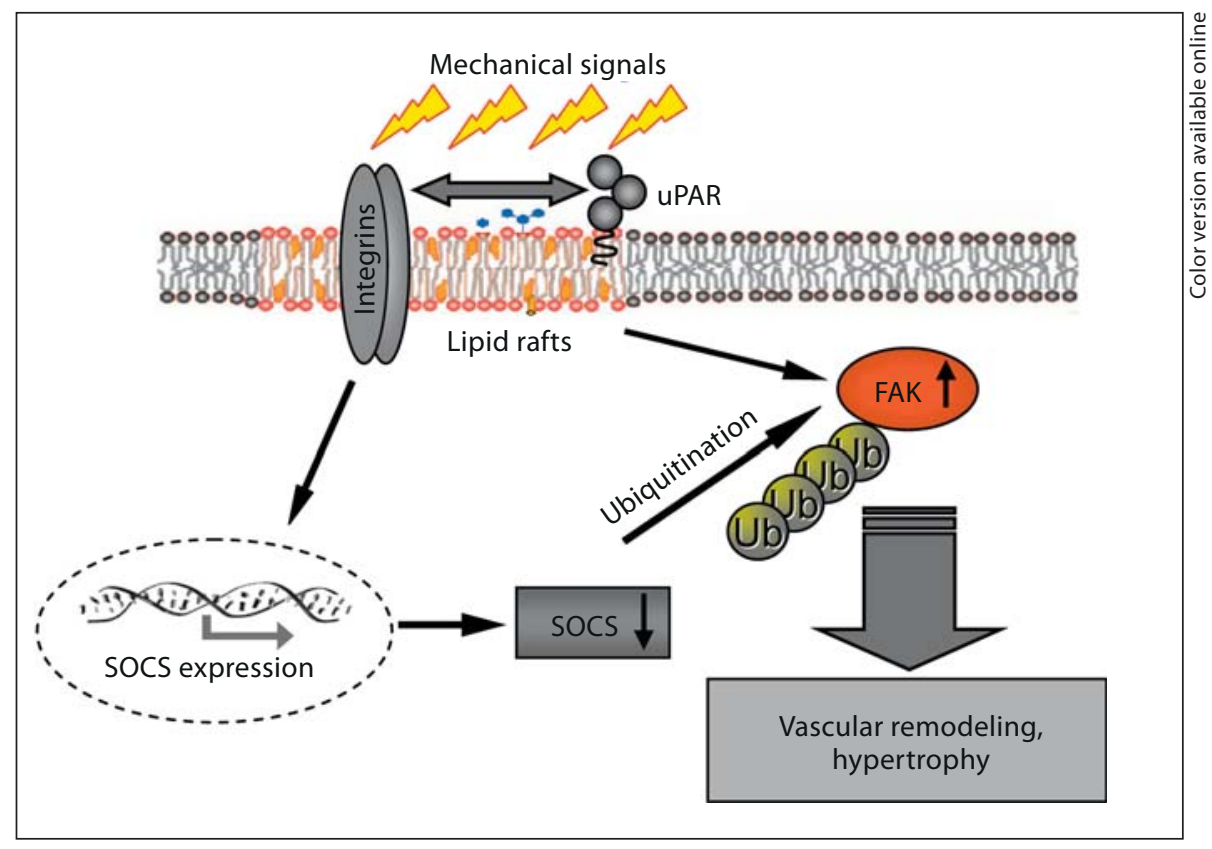

to other stimuli beyond mechanical forces and in other cell types.

Our results indicate that SOCS-1 regulation via integrin-uPAR requires clustering of this complex in lipid rafts. We show that raft disruption in VSMC by membrane cholesterol depletion using MCD led to the abrogation of changes in SOCS-1 in response to mechanical stretch. The cholesterol-enriched membrane microdomains, lipid rafts, play a key role in cell activation by recruiting and excluding specific signaling components of cell surface receptors upon the receptor engagement [13, 20]. Previous studies have demonstrated that uPAR, which is a GPI-anchored protein, could be found in lipid rafts and in nonraft fractions [21]. In this report, we provide evidence that the molecular machinery of uPARand integrin-mediated VSMC response to mechanical stretch employs lipid rafts for signal transduction and cell functional changes. Lipid rafts are required for UPARintegrin association under mechanical stretch resulting in SOCS-1 inhibition. Because we observed that uPAR downregulation abrogated SOCS-1 expression already in control cells, a more profound function for UPAR in SOCS-1 regulation in VSMC might be suggested. uPAR recruitment to lipid rafts initiated by stretch might also have an additional still unknown function beyond regulation of SOCS-1.

FAK, a primary integrin and uPAR effector at focal adhesion sites, is rapidly activated by mechanical stimuli, as shown in different models in vitro and in vivo [22, 23]. The importance of FAK for the regulation of early gene transcription in response to stretch was demonstrated in neonatal rat cardiac myocytes, indicating that this kinase may coordinate signaling pathways involved in the hypertrophic growth induced by mechanical stress [24, 25]. However, the mechanisms responsible for FAK activation by mechanical stress are still unclear. We report here that FAK protein level in VSMC was increased in response to mechanical stretch, although no change in FAK phosphorylation was observed. We further found that the underlying mechanism was related to the SOCS-1-directed impaired polyubiquitination and proteasomal degradation of FAK. These data are in agreement with the studies of others reporting a negative regulation of FAK by SOCS-1 and SOCS-3 in 3T3 fibroblasts, which was mediated via polyubiquitination-induced degradation. The fact that no changes in FAK phosphorylation upon mechanical stretch were observed under our experimental conditions is intriguing. One possible explanation might be that FAK phosphorylation may have an unusual kinetics that remains to be elucidated. It cannot be excluded, however, that upregulation of FAK protein by mechanical stretch is necessary and sufficient to mediate SOCS-1 proteosomal degradation. Phosphorylation-independent functions of FAK have been demonstrated [26]. One further interesting issue requiring future study is a mechanism by which a transient decrease in SOCS-1 mRNA 
causes a long-term protein downregulation. Multiple factors may influence the correlation between mRNA level and protein abundance [for review, see 27]. Investigation of these mechanisms may provide further clue on the role of mechanical stretch in development of vessel pathology.

Overall, our studies identify a novel mechanism for a cross-talk between integrins, uPAR, SOCS-1 and FAK upon propagation of mechanical stimuli in human VSMC. They further imply a regulation of SOCS-1 expression upon mechanical stretch, which differs from the negative feedback loop regulation of a typical cytokine response. These findings have implications for our un- derstanding of how VSMC sense and transmit mechanical stimuli. Mechanistic insights of these data may disclose new pathogenic aspects of mechanical stress in cardiovascular diseases related to elevated blood pressure. Future research is, however, required to prove the concept on freshly isolated cells and to evaluate significance of our study in vivo in an appropriate animal model.

\section{Acknowledgements}

We are grateful to Prof. Harold A. Chapman and Dr. Ying Wei for a325 peptides. This work was supported by grant Du 344/3-1 from the Deutsche Forschungsgemeinschaft.

\section{References}

-1 Zrins SK, Zatina MA, Giddens GP, Gladov S: Shear stress regulation of artery lumen diameter in experimental atherogenesis. J Vasc Surg 1987;5:413-420

12 Dumler I, Weis A, Mayboroda OA, Maasch C, Jerke U, Haller H, Gulba DC: The Jak/Stat pathway and urokinase receptor signaling in human aortic vascular smooth muscle cells J Biol Chem 1998;273:315-321.

>3 Kunigal S, Kusch A, Tkachuk N, Jerke U, Haller H, Dumler I: Monocyte-expressed urokinase inhibits vascular smooth muscle cell growth by activating Stat1. Blood 2003; 102:4377-4383

4 Pan J, Fukuda K, Saito M, Matsuzaki J, Kodama H, Sano M, Takahashi T, Kato T, Ogawa S: Mechanical stretch activates the Jak/ Stat pathway in rat cardiomyocytes. Circ Res 1999;84:1127-1136.

5 Krebs DL, Hilton DJ: SOCS proteins: negative regulators of cytokine signaling. Stem Cells 2001:378-387.

6 Johnston JA: Are SOCS suppressors, regulators, and degraders? J Leukocyte Biol 2004; 75:743-748.

7 Gómez-Guerrero C, López-Franco O, Sanjuán G, Hernández-Vargas P, Sazuki Y, Ortiz-Munoz G, Blanco J, Egido J: Suppressors of cytokine signaling regulate $\mathrm{Fc}$ receptor signaling and cell activation during immune renal injury. J Immunol 2004;172:69696977.

-8 Yoshimura A, Ohishi HMM, Aki D, Hanada $\mathrm{T}$ : Regulation of TLR signaling and inflammation by SOCS family proteins. J Leukocyte Biol 2004;75:422-427.

$\checkmark 9$ Grote K, Bavendiek U, Grothusen C, Flach I, Hilfiker-Kleiner D, H. D, Schieffer B: Stretchinducible expression of the angiogenic factor CCN1 in vascular smooth muscle cells is mediated by Egr-1. J Biol Chem 2004;279: 55675-55681.
10 Grote K, Flach I, Luchtefeld M, Akin E, Holland S, Drexler H, Schieffer B: Mechanical stretch enhances mRNA expression and proenzyme release of matrix metalloproteinase2 (MMP-2) via NAD(P)H oxidase-derived reactive oxygen species. Circ Res 2003;92: e80-e86.

11 Shushakova N, Tkachuk N, Dangers M, Tkachuk S, Park JK, Zwirner J, Hashimoto K, Haller H, Dumler I: Urokinase-induced activation of the gp130/Tyk2/Stat3 pathway mediates a pro-inflammatory effect in human mesangial cells via expression of the anaphylatoxin C5a receptor. J Cell Science 2005; 118:2743-2753.

12 Orr AW, Heimke BP, Blackman BR, Schwartz MA: Mechanisms of mechanotrandsduction. Dev Cell 2006;10:11-20.

13 Golub T, Wacha S, Caroni P: Spatial and temporal control of signaling through lipid rafts. Curr Opin Neurobiol. 2004;14:542-550.

14 Blasi F, Carmeliet P: uPAR: a versatile signalling orchestrator. Nature Rev Mol Cell Biol 2002;3:932-943.

$\checkmark 15$ Liu E, Cote JF, Vuori K: Negative regulation of FAK signaling by SOCS proteins. EMBO J 2003;22:5036-5046.

16 Vuong BQ, Arenzana TL, Showalter BM, Losman J, Chen XP, Mostecki J, Banks AS, Limnander A, Fernandez N, Rothman PB: SOCS-1 localizes to the microtubule organizing complex-associated $20 \mathrm{~S}$ proteasome Mol Cell Biol 2004;24:9092-9101.

17 Shaw A, Xu Q: Biomechanical stress-induced signaling in smooth muscle cells: an update. Curr Vasc Pharmacol 2003;1:41-58.

18 Porter JC, Hogg N: Integrins take partners: cross-talk between integrins and other membrane receptors. Trends Cell Biol 1998;8: 390-396.
19 Chapman HA: Plasminogen activators, integrins, and the coordinated regulation of cell adhesion and migration. Curr Opin Cell Biol 1997;9:714-724.

20 Shaw AS: Lipid rafts: now you see them, now you don't. Nat Immunol 2006;7:1139-1142.

21 Cunningham O, Andolfo A, Santovito ML, Iuzzolino L, Blasi F, Sidenius N: Dimerization controls the lipid rafts partitioning of uPAR/CD87 and regulates its biological functions. EMBO J 2003;22:5994-6003.

22 Franchini KG, Torsoni AS, Soares PHA, Saad MJA: Early activation of the multicomponent signaling complex associated with focal adhesion kinase induced by pressure overload in the rat heart. Circ Res 2000;87: 558-565.

$\checkmark 23$ Laser M, Willey CD, Jiang W, Cooper C, Menick DR, Zile MR, Kuppuswamy D: Integrin activation and focal complex formation in cardiac hypertrophy. J Biol Chem 2000;275: 35624-35630

24 Fonseca PM, Inoue RY, Kobarg CB, CrosaraAlberto DP, Kobarg J, Franchini KG: Targeting to $\mathrm{C}$-terminal myosin heavy chain may explain mechanotransduction involving focal adhesion kinase in cardiac myocytes. Circ Res 2005;96:73-81.

25 Torsoni AS, Constancio SS, Nadruz JW, Hanks SK, Franchini KG: Focal adhesion kinase is activated and mediates the early hypertrophic response to stretch in cardiac myocytes. Circ Res 2003;93:140-147.

26 Kabir J, Lobo M, Zachary I: Staurosporine induces endothelial cell apoptosis via focal adhesion kinase dephosphorylation and focal adhesion disassembly independent of focal adhesion kinase proteolysis. Biochem J 2002;367.

27 Greenbaum D, Colangelo C, Williams K, Gerstein M: Comparing protein abundance and mRNA expression levels on a genomic scale. Genome Biol 2003;4:117-125. 